\title{
Intranasal Ketamine for Refractory Complex Regional Pain Syndrome
}

\author{
Vinita Singh, MD' ${ }^{1}$ Jacob Mills, PharmD, MD², and Kimberly Curseen, MD ${ }^{1}$
}

Complex regional pain syndrome (CRPS) is an often debilitating condition that presents with pain frequently refractory to common treatments. Ketamine has shown promise as a treatment option, particularly intravenous (IV) infusions, but disadvantages include cost and the logistical challenges of multiple repeated infusions (1). Intranasal ketamine has been reported to treat a wide range of conditions including chronic non-cancer pain (2) and neuropathic pain (3). Here we describe the successful off-label use of intranasal ketamine as a maintenance treatment for refractory CRPS.

\section{CASE DESCRIPTION}

A 45-year-old male, with past medical history significant for acute myeloid leukemia in remission status post bone marrow transplant 9 years ago with subsequent onset of graft versus host disease (GVHD), hypertension, and migraines, presented with refractory CRPS in his right upper extremity meeting the Budapest Criteria in sensory, vasomotor, and motor categories. In addition to uncontrolled pain secondary to CRPS he also had left shoulder pain secondary to recurrent acromioclavicular (AC) joint disarticulation and generalized arthralgia related to GVHD. His psychiatric history was significant for post-traumatic stress disorder (PTSD), anxiety, and depression. He had developed symptoms of CRPS in his right upper extremity 5 years ago after an IV line was started for rituximab infusion in his distal forearm. He immediately felt shooting pain followed by

From : 'Department of Anesthesiology, Emory University School of Medicine, Atlanta, GA; ${ }^{2}$ Swain Comprehensive Pain Services, Ashville, NC

Author for correspondence: Vinita Singh, MD

Address: Department of Anesthesiology, Emory University of Medicine, 550 Peachtree Rd NE, Atlanta, GA 30308

E-mail: Vinita.Singh@emory.edu decreased sensation, mainly in the right second and third digits on the dorsum of the hand, in superficial radial nerve distribution. Over the next 2 weeks, his symptoms worsened with shooting and burning pain rated $7 / 10$ on a numerical pain rating scale (NPRS), and a decreased range of motion developed. A right radial nerve block performed 2 weeks after the inciting event provided $100 \%$ pain relief for one day and then his prior symptoms fully returned. The symptoms progressively worsened, with development of limited range of motion, atrophy, and allodynia in the right forearm. The pain was worse with extension and ulnar deviation of the hand. The patient underwent right wrist neuroma resection 3 months after the onset of CRPS, but the symptoms continued to worsen. $\mathrm{He}$ also underwent physical therapy, without any improvement. The patient was not interested in any further injections or procedures, including stellate ganglion block. He had failed antineuropathics such as gabapentin, pregabalin, lamictal, amitriptyline, and venlafaxine, nonsteroidal anti-inflamatory drugs such as diclofenac and celebrex, muscle relaxants such as cyclobenzaprine, topical agents such as lidocaine patches, and opioids such as tramadol and methadone. A buprenorphine patch $20 \mathrm{mcg} / \mathrm{hr}$ was somewhat helpful, but his goal was to reduce opioids due to constipation and "interference with his ability to interact with family."

Monthly IV ketamine infusions (275 mg given over 5-6 hours) were started about 3 years after the onset and provided noticeable improvement in symptoms, but it only lasted 2 weeks, leaving the patient with a portion of each month with worsened pain. About 4 years after the onset, he was then started on intranasal ketamine at $50 \mathrm{mg} 3$ times daily to be delivered via nasal spray containing ketamine $200 \mathrm{mg} / \mathrm{mL}$. He noted significant improvement in functionality and family interactions after starting intranasal ketamine. His neuropathic pain from CRPS of his left upper extremity was controlled with this regimen. He stated 
that he is never pain-free except after his ketamine infusions, but the intranasal ketamine kept most of the symptoms at baseline so he could function. The dose was subsequently titrated to 4 times daily due to a shoulder infection after a distal clavicle resection for recurrent $A C$ joint separation and mucositis from GVHD. Ketamine infusions were continued. At the 9-month follow-up, he continued to report nearly $50 \%$ reduction in pain on a NPRS, with controlled symptoms between infusions. He denies any side effects from intranasal ketamine, but has reported hallucination and feeling of unreality during one of his infusions. He has chronic nausea and fatigue, which he attributes to chronic GVHD.

\section{Comment}

According to the International Association for the Study of Pain, CRPS is a syndrome consisting of continuing regional pain that is disproportionate in intensity or duration to the usual course of any known trauma or lesion. The pain is regional and is associated with abnormal sensory, motor, sudomotor, vasomotor, and/or trophic findings (4). The initial treatments of CRPS are often conservative in nature, combining physical therapy with medications. Refractory pain can be treated with sympathetic nerve blocks (stellate ganglion and lumbar sympathetic block) and even spinal cord stimulators (5).

Ketamine is an anesthetic approved by the Food and Drug Administration, with amnesic, analgesic, dissociative, and sedative properties. It is a noncompetitive, antagonist of $\mathrm{N}$-methyl-D-aspartate (NMDA) receptors that blocks the NMDA channel in the open state by binding to the phencyclidine site located within the lumen of the channel. The antagonism of NMDA receptors produces antinociception of persistent or neuropathic pain in animal models and analgesia in pain states in humans. The NMDA receptor is believed to play a role in the development of opioid tolerance (6). Ketamine is classified as a schedule III medication by the United States Drug Enforcement Agency. Great caution should be taken when using ketamine for pain controls, as its use as a recreational drug has been reported (1).
Ketamine has been used for CRPS with varying success. A 2015 systemic review examining the evidence of ketamine (IV infusions, subcutaneous infusions, and oral) for CRPS concluded there is only weak evidence supporting the efficacy of ketamine for CRPS, but there is clear rationale for its use (2). The most common route of administration of ketamine is intravascular or intramuscular in an inpatient/infusion setting. Although it has been given orally and rectally, the bioavailability of ketamine when given via these routes is only $20-30 \%$ (3). Oral absorption is limited due to the extensive metabolism. Intranasal administration allows higher $(45-50 \%)$ absorption $(7,8)$ when compared to the oral or rectal route, avoids first pass hepatic metabolism, and provides a needle-free, patient-friendly route of administration compared to the IV or intramuscular route. If successful, intranasal ketamine can provide a method of maintenance therapy as an outpatient, without the need for IV access. Intranasal ketamine has been used for multiple medical conditions including depression, PTSD, and breakthrough pain for patients on chronic, long-acting opioids with varying success (2). It also provides a route of administration of ketamine when IV access may be challenging. A retrospective review of intranasal ketamine for prehospital analgesia in cases where vascular access was foreseen or proved hard to establish was described by Johansson et al (9) in 2013. Successful use of intranasal ketamine for pain in the emergency room setting has been described in prospective observational trials by Andolfatto et al (10) and Yeaman et al (11). Other than one patient in the cohort used by Carr et al (2), its documented use for CRPS is lacking.

\section{CONCLUSION}

Here we present a case of intranasal ketamine for chronic maintenance therapy of refractory CRPS. The long-term effects of intranasal ketamine are still unknown, and clinical trials are needed to inform paradigms of clinical treatment of CRPS and other painful conditions using ketamine. 


\section{REFERENCES}

1. Connolly SB, Prager JP, Harden RN. A systematic review of ketamine for complex regional pain syndrome. Pain Med 2015; 16:943-969.

2. Carr DB, Goudas LC, Denman WT, Brookoff D, Lavin PT, Staats PS. Safety and efficacy of intranasal ketamine in a mixed population with chronic pain. Pain 2004; 110:762-764.

3. Huge V, Lauchart M, Magerl W, Schelling G, Beyer A, Thieme D, Azad SC. Effects of low-dose intranasal (S)-ketamine in patients with neuropathic pain. Eur ] Pain 2010; 14:387-394.

4. Harden RN, Oaklander AL, Burton AW, Perez RS, Richardson K, Swan M, Barthel J, Costa B, Graciosa JR, Bruehl S; Reflex Sympathetic Dystrophy Syndrome Association. Complex regional pain syndrome: Practical diagnostic and treatment guidelines, 4th edition. Pain Med 2013; 14:180-229.

5. Eijs FV S-HM, Zundert JV, Faber CG, Lubenow TR, Mekhail N, van Kleef M, Huygen F. Complex regional pain syndrome. In Zundert JV, Patiin J, Hartrick CT, Lataster A, Huygen F, Mekhai $\mathrm{N}$, van Kleef $\mathrm{M}$ (eds). Evidence-based Interventional Pain Practice: According to Clinical Diagnoses. 1st ed. Wiley-Blackwell, Oxford 2012, pp 123-136.

6. Larcher A, Laulin JP, Celerier E, Le Moal M, Simonnet G. Acute tolerance associated with a single opiate administration: Involvement of $\mathrm{N}$-methyl-D-aspartate-dependent pain facilitatory systems. Neuroscience 1998; 84:583-589.

7. Malinovsky JM, Servin F, Cozian A, Lepage JY, Pinaud M. Ketamine and norketamine plasma concentrations after i.v., nasal and rectal administration in children. Br J Anaesth 1996; 77:203207.

8. Yanagihara Y, Ohtani M, Kariya S, Uchino K, Hiraishi T, Ashizawa N, Aoyama T, Yamamura Y, Yamada Y, Iga T. Plasma concentration profiles of ketamine and norketamine after administration of various ketamine preparations to healthy Japanese volunteers. Biopharm Drug Dispos 2003; 24:37-43.

9. Johansson J, Sjöberg J, Nordgren M, Sandström E, Sjöberg $\mathrm{F}$, Zetterström H. Prehospital analgesia using nasal administration of S-ketamine: A case series. Scand J Trauma Resusc Emerg Med 2013; 21:38.

10. Andolfatto G, Willman E, Joo D, Miller P, Wong WB, Koehn M, Dobson R, Angus E, Moadebi S. Intranasal ketamine for analgesia in the emergency department: A prospective observational series. Acad Emerg Med 2013; 20:1050-1054.

11. Yeaman F, Meek R, Egerton-Warburton D, Rosengarten $P$, Graudins A. Sub-dissociative-dose intranasal ketamine for moderate to severe pain in adult emergency department patients. Emerg Med Australas 2014; 26:237-242. 
